[Research Paper]

\title{
$\mathrm{HSV}$ 컬러 모델 및 코너 검출 알고리즘을 이용한 딥러닝 기반의 화염 감지에 관한 연구
}

\author{
류진규 - 곽동걸 ${ }^{* \dagger}$ \\ 강원대학교 방재전문대학원 대학원생, "강원대학교 방재전문대학원 교수
}

\section{Flame Detection Based on Deep Learning Using HSV Color Model and Corner Detection Algorithm}

\author{
Jin-Kyu Ryu - Dong-Kurl Kwak ${ }^{* \dagger}$ \\ Graduate Student, Graduate School of Disaster Prevention, Kangwon National Univ., \\ *Professor, Graduate School of Disaster Prevention, Kangwon National Univ.
}

(Received March 8, 2021; Revised March 8, 2021; Accepted March 30, 2021)

\section{요 약}

최근 딥러닝 기법을 이용한 이미지 분류 모델이나 객체 감지 모델이 많이 연구되고 있지만 적절한 전처리 방법을 설계하지 않을 경우 성능 평가 결과 낮은 정확도를 얻을 수 있다. 따라서 본 연구에서 제안하는 효과적인 화염검출 전처리 방법으로는 HSV 컬러 모델과 Harris 코너 검출 알고리즘을 적용한 이미지 전처리 방법이다. HSV 컬러 모델 을 통해 화염이 존재하는 색상영역을 필터링하고, 필터링된 결과물에 대해 Harris 코너 검출 방법을 적용할 경우 화 염 이미지의 거친 질감 특성 때문에 화염 주변에 집중적으로 코너가 검출되게 된다. 이러한 특성을 통해 코너가 다수 발생한 영역을 관심영역으로 검출하여 딥러닝 기반의 합성곱신경망(Convolutional neural network, $\mathrm{CNN}$ ) 모델을 통해 최종적으로 화염 여부를 분류하도록 하였다. 그 결과 본 연구에서 제안한 모델의 화염 검출 결과 정확도는 $97.5 \%$ 정밀도는 $97 \%$ 로 나타났다.

\section{ABSTRACT}

Recently, many image classification or object detection models that use deep learning techniques have been studied; however, in an actual performance evaluation, flame detection using these models may achieve low accuracy. Therefore, the flame detection method proposed in this study is image pre-processing with HSV color model conversion and the Harris corner detection algorithm. The application of the Harris corner detection method, which filters the output from the HSV color model, allows the corners to be detected around the flame owing to the rough texture characteristics of the flame image. These characteristics allow for the detection of a region of interest where multiple corners occur, and finally classify the flame status using deep learning-based convolutional neural network models. The flame detection of the proposed model in this study showed an accuracy of $97.5 \%$ and a precision of $97 \%$.

Keywords : Artificial intelligence, Deep learning, HSV color model, Corner detection algorithm, CNN

\section{1. 서 론}

소방청 국가화재정보센터에서 공개한 발화요인별 전국 화재 발생건수 통계에 따르면 2020년 기준 부주의에 의한 화재가 19,177 건으로 가장 많았고, 전기적 요인이 9,310건, 기계적 요인이 4,054건 발생한 것으로 나타났다. 특히 이 중 전기화재에서 발생하는 인명피해의 경우 사고사례의 절반
이상이 일반 주거시설에서 발생함에 따라 옥내용 감지기 시 설 등의 고도화 및 정밀화가 필요하다. 하지만 기존 재래식 감지기의 경우 비화재보와 같은 오동작 등의 신뢰성 문제가 꾸준히 제기되고 있어 딥러닝 기반의 인공지능과 같은 최첨 단 기법들을 이용한 화재감지 연구가 필요한 실정이다 ${ }^{(1)}$.

본 연구에서는 이미지 전처리 및 딥러닝 기반의 화재 감 지를 위해 Figure 1과 같은 이미지 기반의 전처리 과정을

Corresponding Author, TEL: +82-33-570-6823, FAX: +82-33-570-6301, E-Mail: dkkwak@kangwon.ac.kr

(c) 2021 Korean Institute of Fire Science \& Engineering. All right reserved. 


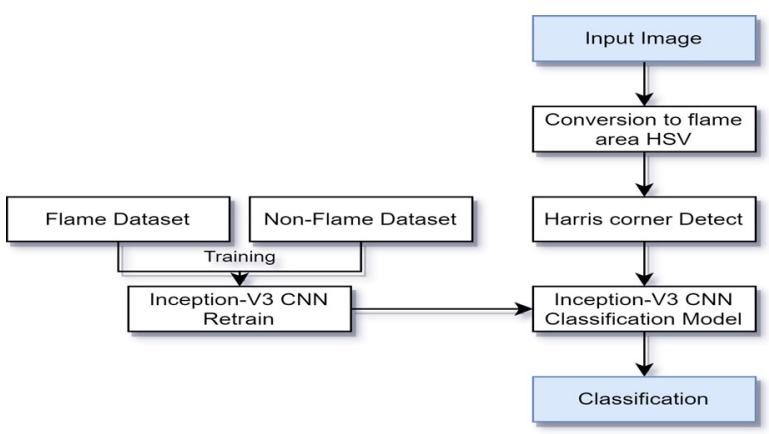

Figure 1. Imge processing flow diagram for video based fire detection.

수행한다. 카메라를 통해 입력된 이미지 내에는 화염에 해 당하는 객체 이외에도 여러 객체가 존재하므로 딥러닝 기 반의 이미지 분류모델을 수행하기 위해서는 이미지 전처리 가 필수적이다. 따라서 먼저 입력 영상의 이미지에 대해 전 처리를 하여 화재와 관련된 화염일 가능성이 높은 관심영 역(Region of Interest, RoI)을 검출하도록 한다. 이를 위해 본 연구에서는 HSV 컬러 변환을 수행하도록 하고, Harris corner detector 알고리즘을 이용한다. 그리고 필터링되고 남 은 영역에 대해서는 Inception-V3 합성곱신경망 모델을 이 용하여 화염 및 비화염 여부를 추론하도록 한다. 이때 Inception-V3 모델은 수집된 약 1만여 장의 화염 이미지 데 이터셋을 이용하여 학습을 진행하도록 하였다.

\section{2. 이미지 전처리 방법 설계}

\subsection{HSV 컬러 변환}

본 연구에서는 첫 번째 이미지 전처리로 입력 이미지에 대해 HSV 컬러 변환을 하였다. HSV 컬러 모델의 색상(Hue) 과 채도(Saturation)성분은 인간이 색상을 인식하는 방법과 유사한 방법으로 색상의 특징들을 다룰 수 있으므로 이미지 전처리 이외에도 여러 응용분야에서 객체들의 색상을 식별 하는데 사용할 수 있어 색상 특성에 기반한 영상처리 알고 리즘의 개발을 위한 이상적인 추출 도구로 사용할 수 있다.

$\mathrm{HSV}$ 컬러 모델에서 색상에 해당하는 Hue는 파장이 가 장 긴 빨간색을 기준으로하는 색상의 분포를 나타내며, 채 도 성분인 Saturation은 순수한 색상에 하얀 빛이 포함된 정 도를 나타낸다. 또한 명도에 해당하는 Value는 빛의 강도를 측정하는 정도로 사용된다. Value가 하나의 성분으로 독립 되어 범위를 조절할 수 있으므로 조명 변화에 강인한 알고 리즘을 생성할 수 있다 ${ }^{(2,3)}$.

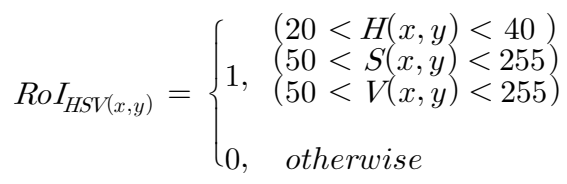

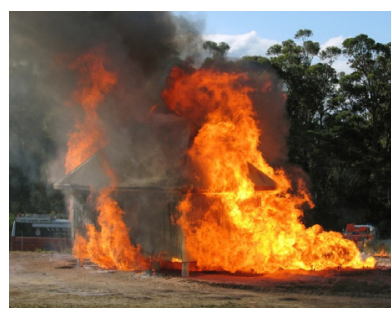

(a) Original image

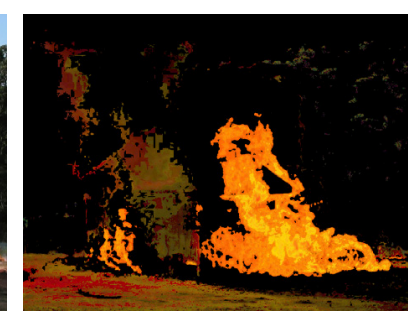

(b) converted image
Figure 2. Comparison of original image and HSV color converted image.

식 (1)에서 $R_{H} I_{H S V(x, y)}$ 는 지정된 컬러 모델의 범위에 해 당하는 픽셀의 좌표이며 $H(x, y)$ 및 $S(x, y), V(x, y)$ 는 각각 이미지의 픽셀 $(x, y)$ 위치에서 화염이 존재할 수 있는 색공 간에 해당되는 영역을 의미한다. 지정된 범위에 해당되는 이미지는 첫 번째 이미지 전처리의 결과물로 추출되게 되 고, 픽셀값이 0 인 것은 비화염 색상영역으로 분류된 픽셀 을 의미한다. 이러한 HSV 컬러 변환의 결과를 보여주는 것 이 Figure 2로 (a)에 해당되는 것이 원본 화염 이미지이고, (b)는 HSV 컬러 변환이 적용된 결과물에 대한 이미지이다.

\subsection{Harris corner detector}

$\mathrm{HSV}$ 컬러 변환을 한 이후에도 화염 이외의 나뭇잎이나 연한 노랑색을 포함한 객체 등의 결과물이 남을 수 있다. 이러한 불필요한 객체들을 추가적으로 필터링하고자 두 번 째 이미지 전처리로 Harris corner detector를 사용하였다. $\mathrm{HSV}$ 컬러 변환을 하고 남은 결과 객체들 중 화염의 경우 텍스쳐의 질감이 거친 특성이 있어 코너점이 다수 발생되 는 특징이 있다. 이를 통해 HSV 컬러 변환된 결과 이미지 에 대해 Harris corner detector를 수행하면 화염부에만 집중 적으로 코너점이 발생하여 좀 더 정밀하게 필터링을 수행 할 수 있게 된다.

Harris corner detector를 수행하기 위해 먼저 이미지에서 기준점 $(x, y)$ 가 있을 때 기준점으로부터 $(u, v)$ 만큼 이동한 변화량을 가진다고 가정하면, 변화량 $E(x, y)$ 는 식 (2)와 같 이 표현할 수 있다.

$$
\begin{aligned}
& E(x, y) \\
& =\Sigma_{W}\left[I\left(x_{i}, y_{i}\right)-I\left(x_{i}+u, y_{i}+v\right)\right]^{2}
\end{aligned}
$$

여기서 $I$ 는 밝기를 나타내며, $\left(x_{i}, y_{i}\right)$ 는 가우시안 윈도우 $W$ 내부의 점들을 나타낸다. $(u, v)$ 만큼 움직인 영역을 테일 러 급수를 이용하면 식 (3)과 같이 정리된다.

$$
\begin{aligned}
& I\left(x_{i}+u, y_{i}+v\right) \approx \\
& I\left(x_{i}, y_{i}\right)+\left[I_{x}\left(x_{i}, y_{i}\right) I_{y}\left(x_{i} y_{i}\right)\right]\left[\begin{array}{l}
u \\
v
\end{array}\right]
\end{aligned}
$$

$I_{x}$ 및 $I_{y}$ 는 각각 $x$ 와 $y$ 방향의 1 차 미분으로 Figure 3 과 같 


$S_{x}=$\begin{tabular}{|l|l|l|}
\hline-1 & 0 & 1 \\
\hline-2 & 0 & 2 \\
\hline-1 & 0 & 1 \\
\hline
\end{tabular}

$S_{y}=$\begin{tabular}{|c|c|c|}
\hline 1 & 2 & 1 \\
\hline 0 & 0 & 0 \\
\hline-1 & -2 & -1 \\
\hline
\end{tabular}

Figure 3. Masks used by sobel filter.

은 소벨 $x$ 커널인 $S_{x}$ 와 소벨 $y$ 커널인 $S_{y}$ 를 이용한 합성곱을 통해 각각 구할 수 있다. 그리고 식 (3)을 식 (2)에 대입하 면, 전체 변화량 $E(u, v)$ 는 식 (4)와 같이 나타낼 수 있다.

$$
\begin{aligned}
& E(u, v) \\
& =\left[\begin{array}{ll}
u & v
\end{array}\right]\left[\begin{array}{cc}
\sum_{W}\left(I_{x}\left(x_{i}, y_{i}\right)\right)^{2} & \sum_{W} I_{x}\left(x_{i}, y_{i}\right) I_{y}\left(x_{i}, y_{i}\right) \\
\sum_{W} I_{x}\left(x_{i}, y_{i}\right) I_{y}\left(x_{i}, y_{i}\right) & \sum_{W}\left(I_{y}\left(x_{i}, y_{i}\right)\right)^{2}
\end{array}\right]\left[\begin{array}{l}
u \\
v
\end{array}\right] \\
& =\left[\begin{array}{ll}
u & v
\end{array}\right]\left[\begin{array}{l}
u \\
v
\end{array}\right]
\end{aligned}
$$

여기서 $M$ 은 전체 변화량의 행렬로써, $M=\left[\begin{array}{ll}A & C \\ C & B\end{array}\right]$ 이라 한다면 행렬 $\mathrm{M}$ 에서 두 값은 식 (5)와 (6) 같은 성질을 만 족한다. 그리고 최종적으로 식 (7)을 통해 에지, 코너, 평면 을 결정할 수 있다. $k$ 는 경험적 상수로서 본 논문에서는 0.04 의 값을 사용하였다.

$$
\begin{aligned}
& \operatorname{det}(M)=A B-C=\lambda_{1} \lambda_{2} \\
& \operatorname{trace}(M)=A+B=\lambda_{1}+\lambda_{2} \\
& R(x, y)=\operatorname{det}(M)-k(\operatorname{trace}(M))^{2}
\end{aligned}
$$

픽셀의 위치마다 $M$ 의 값이 달라지며, 최종적으로 계산 하여 나온 고유값 $R(x, y)$ 는 다음 조건에 비교하여 에지, 코 너, 평면을 구분하게 된다(4-7).

- $|R|$ 이 작고, $\lambda_{1}$ 과 $\lambda_{2}$ 가 모두 작을 때 해당 영역은 평 면이다.

- $R<0$ 인 조건이고 $\lambda_{1} \gg \lambda_{2}$ 이거나 반대인 경우 해당 영역은 에지이다.

- $R$ 이 큰 값을 가질 경우 해당 영역은 코너이다.

조건들 중 본 연구에서 필요한 조건에 해당하는 것은 $R$ 이 큰 값을 가져 코너점에 해당하는 경우로 Figure 4는 해당 조건을 만족하는 픽셀을 시각화한 결과물이다. Figure 4(a) 는 이미지 내에 화염이 존재하지 않는 샘플의 전처리결과 이고, (b)는 화염이 존재하는 샘플의 전처리결과로 HSV 컬 러 변환된 결과 이미지에서 코너점 조건을 만족하는 픽셀 들은 녹색 점으로 표시하도록 하였다.

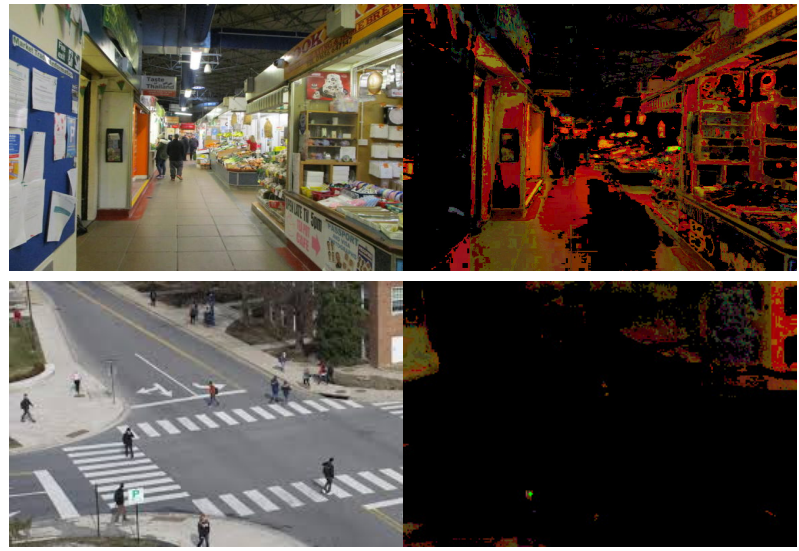

(a) HSV color converted Non-flame images
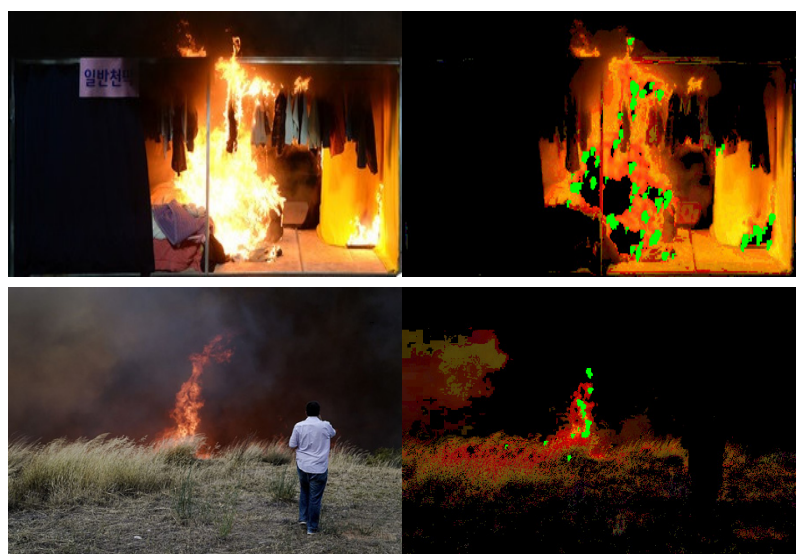

(b) HSV color converted flame images

Figure 4. Comparison of original and corner detection result images.

비화염 샘플의 경우 화염이 아닌 객체임에도 HSV 컬러 변환으로도 필터링되지 않은 픽셀들이 다수 존재하지만 코 너점 검출까지 수행하였을 경우 대부분 코너점이 존재하지 않는 결과를 확인할 수 있다.

화염이 존재하는 샘플의 경우는 화염에 해당하지 않는 객체들이 HSV 컬러 변환으로 완벽히 필터링이 안되었지 만, 화염이 존재하는 영역에서는 코너점들이 집중적으로 검출되는 결과를 확인할 수 있다. 따라서 이러한 결과를 통 하여 이미지 내에 다양한 객체들이 존재하는 경우 효과적 으로 전처리를 수행할 수 있다. 그리고 코너점들이 밀집하 여 발생한 영역을 딥러닝 기반의 합성곱신경망을 통해 추 론할 관심영역으로 사용하도록 한다.

\section{3. 딥러닝 기반의 합성곱신경망 설계}

딥러닝 기반의 합성곱신경망을 이용한 이미지 학습을 할 때, 깊은 계층과 넓은 노드로 구성하여 사용한다면 더 높은 정밀도를 얻을 수 있는 것이 일반적이다. 하지만 이렇 게 구성할 경우 파라미터의 양이 증가해 연산량이 상당히 


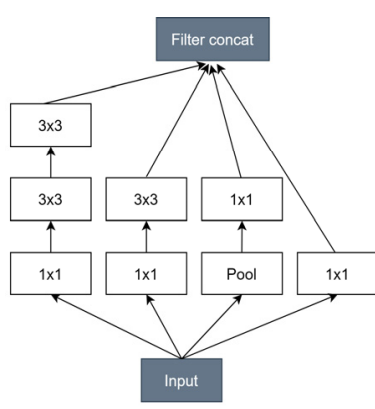

(a) Inception A module

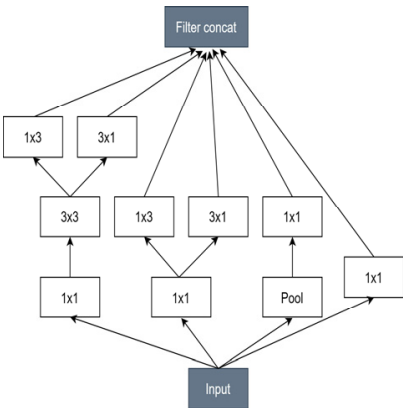

(c) Inception $\mathrm{C}$ module

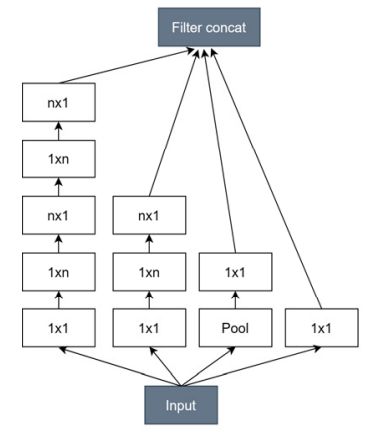

(b) Inception B module

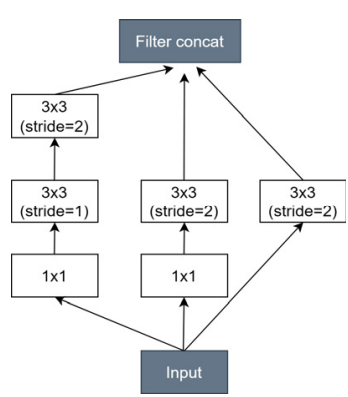

(d) Reduction module
Figure 5. Structure of inception modules.

늘어나고, 과적합(Over fitting) 문제나 기울기 소실(Gradient vanishing problem)이 발생하게 된다. 따라서 노드간의 연결 은 간결(Sparse)하게 하고, 행렬연산은 밀집(Dense)되도록 하 여야 한다. 이를 반영하여 전체적인 신경망은 깊으면서도 연 산에 어려움이 없도록 만든 것이 Inception 구조이다 ${ }^{(8-10)}$

Figure 5의 (a)는 Inception A, (b)는 Inception B 그리고 (c) 는 Inception $\mathrm{C}$ 모듈의 구조를 보여주고 있는데, 특징적인 것으로는 $1 \times 1$ 컨볼루션 필터가 포함되어 있는 것이다.

$1 \times 1$ 필터는 평면상에서 컨볼루션을 수행하더라도 높 이나 넓이의 변화가 없으므로 공간적인 특징 손실은 없다. 따라서 이 필터의 역할은 여러 계층의 합성곱 수행으로 인 해 채널 수가 커지는데, 이 채널의 수를 조절하는 기능을 한다. 이로 인하여 $1 \times 1$ 필터 다음에 나오는 $3 \times 3$ 이나 5 $\times 5$ 필터에서의 파라미터 개수를 절약할 수 있다. 따라서 Inception-v3 모델은 다른 $\mathrm{CNN}$ 모델들보다 더욱 계층이 깊 지만 파라미터는 비교적 크지 않은 장점을 갖는다.

Table 1은 Inception 모듈들을 이용하여 구성한 $\mathrm{CNN}$ 계 층의 구성을 나타내고 있다. 입력 이미지 크기의 경우 299 $\times 299$ 로 설정되었고, 일반적인 합성곱신경망에서는 파라미 터의 크기를 줄이고자 모듈 또는 계층 사이에 Pooling을 사용 하였으나, 이미지의 특징 손실이 커지는 Representational bottleneck 문제가 발생하므로 이를 해결하기 위해 Figure 5 의 (d)와 같은 파라미터 규모를 축소시키는(Dimmension reduction)모듈을 이용하여 차원을 축소하는 방법을 사용하 였다.
Table 1. Inception-V3 CNN Parameter

\begin{tabular}{c|c|c}
\hline Layer & Kernel size & Input size \\
\hline \hline Conv & $3 \times 3$ & $299 \times 299 \times 3$ \\
\hline Conv & $3 \times 3$ & $149 \times 149 \times 32$ \\
\hline Conv (padding) & $3 \times 3$ & $147 \times 147 \times 32$ \\
\hline MaxPool & $3 \times 3$ & $147 \times 147 \times 64$ \\
\hline Conv & $3 \times 3$ & $73 \times 73 \times 64$ \\
\hline Conv & $3 \times 3$ & $73 \times 73 \times 80$ \\
\hline MaxPool & $3 \times 3$ & $71 \times 71 \times 192$ \\
\hline Inception Ax3 & As in Fig. 5 (a) & $35 \times 35 \times 192$ \\
\hline Reduction & As in Fig. 5 (d) & $35 \times 35 \times 228$ \\
\hline Inception Bx4 & As in Fig. 5 (b) & $17 \times 17 \times 768$ \\
\hline Reduction & As in Fig. 5 (d) & $17 \times 17 \times 768$ \\
\hline Inception Cx2 & As in Fig. 5 (c) & $8 \times 8 \times 1280$ \\
\hline AveragePool & $8 \times 8$ & $8 \times 8 \times 2048$ \\
\hline Fc & - & $1 \times 2048$ \\
\hline Sigmoid & - & 2 \\
\hline
\end{tabular}

마지막으로 최종 계층에서의 활성함수(Activation function) 는 화염 또는 비화염 두 가지에 대한 분류 문제이므로 sigmoid를 사용하였다.

학습을 위해 사용된 이미지 데이터셋은 총 20,177 장이 수집되었으며, 이 중 화염에 관련된 데이터셋은 10,153 장, 비화염에 해당하는 이미지 데이터셋은 10,024 장 수집되었 다. 그리고 각각 학습용 데이터셋(Train dataset)과 검증용 데이터셋(Test dataset)을 8 대 2 비율로 나누어 학습을 진행 하였다.

실험환경으로 CPU는 Intel i7-8700을 사용하였고, GPU는 RTX3070을 사용하였으며, 사용 OS는 Linux Ubuntu 18.04 환경에서 학습 및 테스트를 진행하였다.

Figure 6은 구성된 $\mathrm{CNN}$ 모델의 화염 학습 결과를 나타 내는 것으로 (a)는 정확도(Accuracy)와 (b)는 손실(Loss)을 나타낸다. 빨간색 곡선은 학습용 데이터셋으로, 학습에 사 용된 화염 이미지들 중 오직 학습에만 사용된 데이터셋을 통해 분류한 정확도와 오차인 손실을 나타낸다. 녹색 곡선 은 검증용 데이터셋의 결과로 학습용 데이터셋과는 다르게 학습 과정에 사용되지 않은 데이터셋으로 오직 모델의 정 확도와 손실의 평가 목적으로만 사용된 데이터셋이다. 따 라서 학습용 데이터셋에서는 높은 정확도와 낮은 손실을 보이는데 검증용 데이터셋에서는 비교적 낮은 정확도와 높 은 손실을 보인다면 과적합(Over fitting)이 발생한 것으로 볼 수 있고, 학습이 제대로 진행되지 않은 경우에 해당한 다. 하지만 본 연구의 학습 결과는 두 가지 데이터셋 모두 비슷하게 높은 정확도와 낮은 손실을 보이므로 화염에 대 해 학습이 잘 진행된 것으로 판단할 수 있다. 그리고 일정 이상 정확도가 증가하지 않고, 손실도 더 이상 크게 감소하 


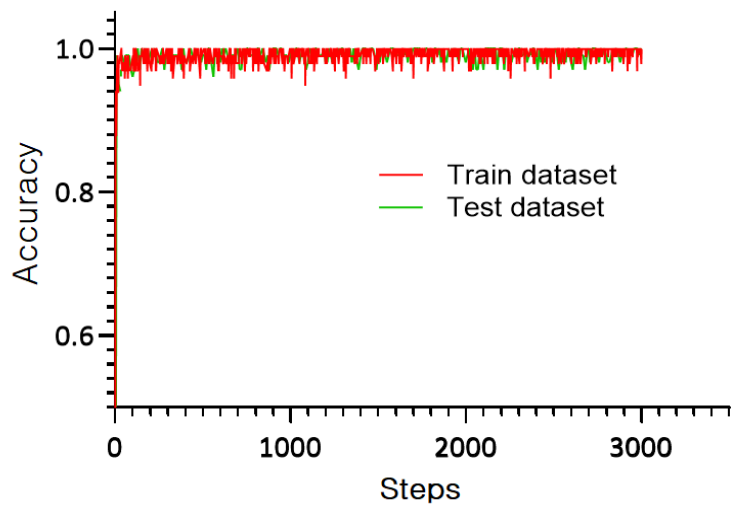

(a) Accuracy curve

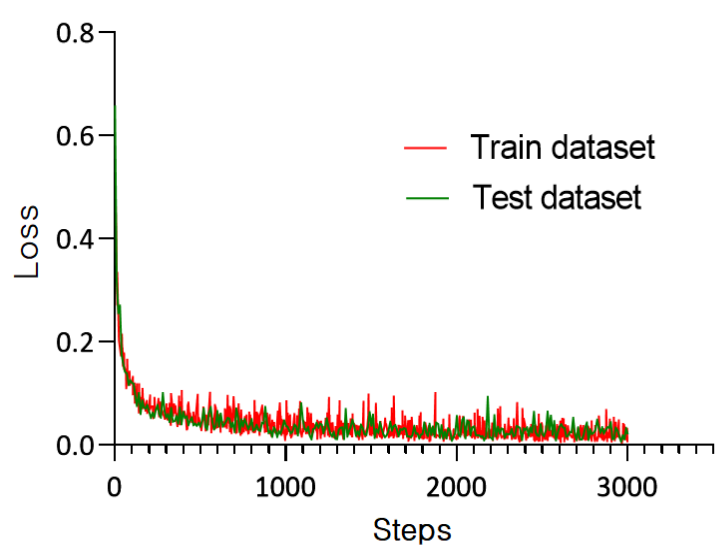

(b) Loss curve

Figure 6. Learning results accuracy and loss curve.

지 않는 학습 횟수인 3천 회에서 학습을 종료하였다.

\section{4. 실험결과 및 성능평가}

Figure 7은 화염 검출 평가 결과를 보여주는 것으로, 제 안하는 화염 감지 알고리즘의 구체적인 성능평가를 위해 직접 촬영한 화재실험 사진과 실제 화재 현장에서 촬영한 사진들을 이용하였고, 주간의 실내, 실외 그리고 야간에 촬 영된 실내, 실외 등 다양한 평가용 사진들로 구성하였다. 제시된 사진은 모두 화염 이미지에 대해 본 연구에서 제시 한 $\mathrm{CNN}$ 모델 및 딥러닝 기반의 객체검출 알고리즘인 Faster R-CNN (Region based CNN)과 Single shot multibox detector (SSD)를 이용하여 추론한 결과를 경계상자로 표시하여 나 타낸 것이다. Figure 7의 좌측부터 (a)는 본 연구에서 제시 한 방법을 통해 검출한 결과로 붉은색 경계상자 부분이 최 종 화염으로 추론한 결과이다. 그리고 (b)는 Faster R-CNN, (c)는 $\mathrm{SSD}$ 를 이용한 검출결과를 보여주고 있으며, 초록색 경계상자가 화염, 하늘색 경계상자가 비화염으로 분류한 결과를 시각화 한 것이다.

본 연구에서 제시한 모델의 경우 화염이 차지하는 객체 의 크기가 작아도 대부분 검출되는 특성을 보였으며, Faster
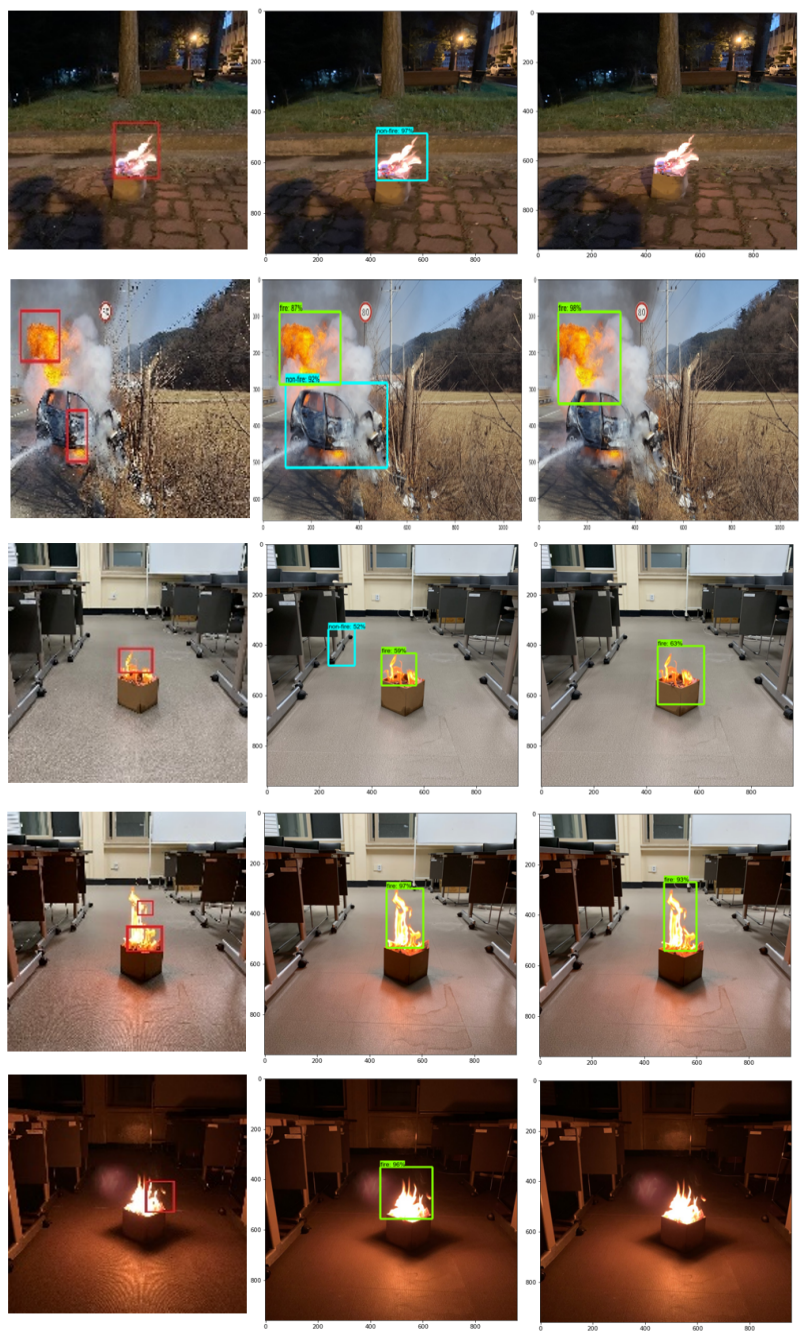

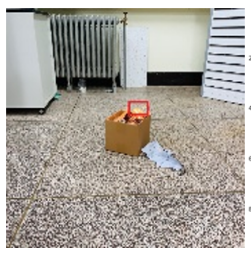

(a) Our proposed

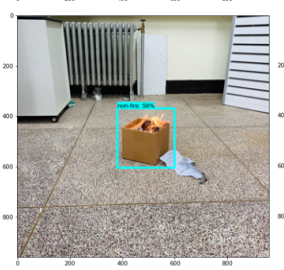

(b) Faster R-CNN

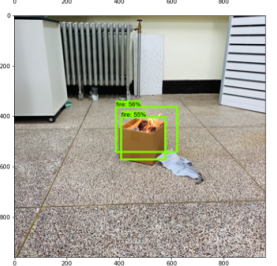

(c) SSD
Figure 7. Flame detection results comparing different models.

$\mathrm{R}-\mathrm{CNN}$ 의 경우도 대부분의 화염에 대해 올바르게 판단하 였지만 일부의 경우는 화염이 아닌 객체로 잘못 판단하기 도 하였다. $\mathrm{SSD}$ 의 경우에는 Faster R-CNN보다 연산시간이 더 적게 소요되었지만 사진 내에서 객체의 크기가 작은 경 우 검출되지 않는 경우가 많이 발생하였다.

테스트 이미지는 화재 및 비화재 사진 각각 100장을 이 용하여 화염 검출을 진행하였으며, 구체적이고 객관적인 정확도 평가를 위해 다음의 식을 통해 검출 결과를 평가하 였다 ${ }^{(11)}$. 


$$
\text { Accuracy }=\frac{T P+T N}{T P+F N+F P+T N}
$$

$$
\begin{aligned}
& \text { Precision }=\frac{T P}{T P+F P} \\
& \text { Recall }=\frac{T P}{T P+F N}
\end{aligned}
$$

$$
F 1 \_S c o r e=2 \cdot \frac{\text { Precision } \cdot \text { Recall }}{\text { Precision }+ \text { Recall }}
$$

True positive (TP)는 화염을 올바르게 검출한 경우에 해 당되고, False negative $(\mathrm{FN})$ 은 화염 이미지로부터 검출을 못한 경우, True negative (TN)은 화염이 아닌 객체에 대해 서 화염이 아닌 것으로 올바르게 검출한 경우, 마지막으로 False positive (FP)는 화염이 아닌 객체에 대해

화염으로 잘못 검출한 경우에 해당한다. 식 (8)은 정확도 (Accuracy)에 해당되는 식으로 화염과 비화염을 옳게 분류 한 경우에 전체 경우를 나눈 결과 값이다. 식 (9)는 정밀도 (Precision)에 대한 계산식으로 옳게 검출 한 횟수 TP에 $\mathrm{FP}$ 까지 더해서 나눈 식이다. 하지만 객체검출이나 이미지분 류 모델에서는 정확도와 정밀도만 두고 성능을 평가하는 것은 적절하지 않다. 따라서 추가적으로 식 (9)의 정밀도와 식 (10)의 검출율(Recall)을 이용하여, 성능율 F1_스코어를 계산하였다.

계산 결과 정확도는 $97.5 \%$, 정밀도는 $97.0 \%, \mathrm{~F} 1$ 스코어 는 97.5\%로 나타났다. 이 결과를 Table 2를 통해 Faster $\mathrm{R}-\mathrm{CNN}$ 및 $\mathrm{SSD}$ 와 비교하여 나타내었고, 비교된 객체검출 모델들은 동일한 이미지 데이터셋을 통해 학습이 되었고, 동일한 테스트 이미지를 이용하여 성능을 평가하였다.

Figure 8은 세 가지 객체검출모델의 구체적인 정확도 비 교를 위한 Receiver operating characteristic (ROC) 커브와 Precision recall (PR) 커브를 보여주고 있다. 두 가지 커브 모두 이진 라벨 분류에서 분류 정확도를 명확하게 시각화 할 수 있는 방법이다.

ROC 커브는 True positive rate (TPR)과 False positive rate (FPR) 두 가지 매개변수를 통해 표현되고, 임계값 기준을 변화시키며 ROC곡선을 나타낸다. 이때 분류임계값을 낮추 면 일반적인 분류모델에서는 양성으로 분류되는 TPR과 $\mathrm{FPR}$ 이 같이 증가하게 되므로 그래프상에서 TPR이 높으면 서 $\mathrm{FPR}$ 이 낮은 커브일수록 분류가 잘되는 모델로 판단할 수 있다. PR 커브도 마찬가지로 임계값의 변화에 의한 분 류모델의 성능을 평가하는 방법으로 Precision과 Tecall 값 이 모두 높을수록 좋은 모델로 판단할 수 있다 ${ }^{(12,13)}$.

비교적 최근의 딥러닝기반의 객체검출 알고리즘인 Faster $\mathrm{R}-\mathrm{CNN}$ 과 $\mathrm{SSD}$ 모델 모두 정확도에서는 큰 차이를 보이지 않았지만, Faster R-CNN의 경우 본 연구 환경에서 장 당 약
Table 2. Performance Comparison for Object Detection Algorithms with the Same Dataset

\begin{tabular}{c|c|c|c}
\hline Model name & Accuracy & Precision & F1-Score \\
\hline \hline Our proposed & $97.5 \%$ & $97.0 \%$ & $97.5 \%$ \\
\hline Faster R-CNN & $88.7 \%$ & $89.4 \%$ & $97.0 \%$ \\
\hline SSD & $79.1 \%$ & $77.8 \%$ & $95.0 \%$ \\
\hline
\end{tabular}

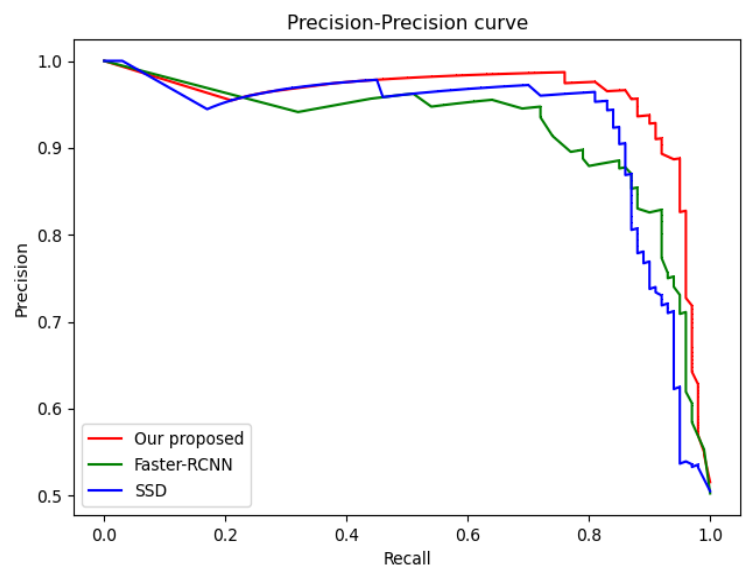

(a) Comparison of PR curves

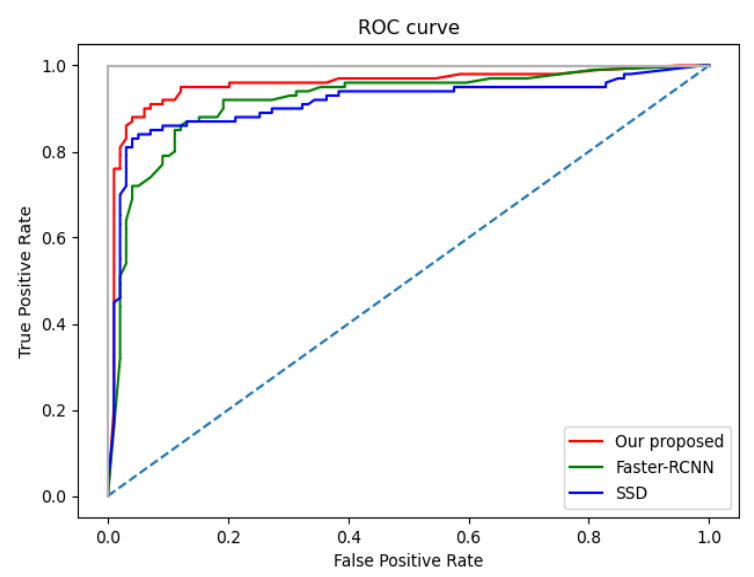

(b) Comparison of ROC curves

Figure 8. ROC and PR curve comparison.

$1.2 \mathrm{~s}$ 소요되었으며, $\mathrm{SSD}$ 의 경우는 $0.32 \mathrm{~s}$ 정도로 속응성 측 면에서는 $\mathrm{SSD}$ 가 우수하나 이미지 내에서 작은 객체에 대해 서는 검출하지 못하는 문제가 있었다. 이는 Faster R-CNN의 경우 일정 크기 및 종횡비를 갖는 Anchor box를 전체 이미 지영역에 대해 탐색하는 방식으로 객체가 존재할 후보영역 을 추출하고, $\mathrm{SSD}$ 의 경우도 이와 유사하게 사전에 설정된 크기를 갖는 검출기를 이동시키며 객체 후보를 추출하므 로, 설정된 검출기보다 작은 경우 객체를 검출하지 못하는 결과를 가져왔다.

그리고 본 연구에서 제시한 화염검출 모델의 경우는 관 심영역을 탐색하는 계층에서의 지연시간이 적어 최종 
$\mathrm{CNN}$ 을 이용한 추론까지 장 당 평균 $0.38 \mathrm{~s}$ 정도가 소요되 었고, 이미지 내에서 객체의 크기에 관계없이 화염으로 추 측되는 객체들은 모두 관심영역으로 추출되었다.

따라서 본 연구에서 제시된 모델의 경우 속응성은 다른 모델과 크게 다르지 않았지만 정확도 및 검출율 부분에서 상당히 효과적으로 향상되었고, 이는 화염일 가능성이 큰 특징 검출을 통해 사전에 화염이 아닌 객체들이 필터링되 어 비교 대상으로 제시된 다른 모델보다 $\mathrm{FP}$ 의 비중이 낮으 면서 $\mathrm{TP}$ 의 비율이 높아 정밀도와 검출율이 모두 향상될 수 있었다.

\section{5. 결 론}

본 연구를 통해 화염검출에 용이한 이미지 전처리방법 을 적용하고 이를 통해 추출된 관심영역에 대하여 딥러닝 기반의 모델을 통한 추론을 진행하였다. 최근 연구되는 객 체검출 기법들의 경우 전체 이미지 영역으로부터 객체가 있을 가능성이 높은 영역을 탐색하기 위해 일정 크기 및 비율을 갖는 검출기를 이동시키면서 발생하는 큰 연산과 객체의 크기가 너무 크거나 작을 경우 검출하지 못하는 문 제점이 있었으나, 본 연구에서 제시한 전처리방법을 이용 할 경우 화염의 크기가 비교적 작아도 검출이 가능한 장점 이 있었다. 또한 이미지 전처리 기법의 경우 알고리즘들을 적절히 사용하여 화염 검출에 용이하도록 설계하여 높은 검출율과 정확도 향상을 확인할 수 있었다.

\section{References}

1. S. W. Jee, S. K. Kim, S. H. Yang, J. J. Lee, P. Y. Kim and C. H. Lee, "Detection of Fire Location And Reliability Improvement of the Conventional Fire Detector and P-type Receiver," Journal of Korean Institute of IIluminating and Electrical Installation Engineers, Vol. 25, No. 5, pp. 39-44 (2011). https://doi.org/10.5207/JIEIE.2011.25.5.039.

2. Z. Yang, W. Shi, Z. Huang, Z. Yin, F. Yang and M. Wang, "Combining Gaussian Mixture Model and HSV Model with Deep Convolution Neural Network for Detecting Smoke in Videos", International Conference on Communication Technology, pp. 1266-1270, (2018). https://doi.org/10.1109/ICCT.2018.8599905.

3. N. Hassanm K. W. Ming and C. K. Wah, "A Comparative Study on HSV-based and Deep Learning-based Object Detection Algorithms for Pedestrian Traffic Light Signal Recognition", International Conference on Intelligent Autonomous Systems, pp.
71-76 (2020). https://doi.org/10.1109/ICoIAS49312.2020.9081854.

4. C. Harris and M. Stephens, "A Combined Corner and Edge Detector”, Alvey Vision Conference, pp. 147-151 (1988). https://doi.org/10.5244/c.2.23.

5. P. Rakshit, D. Bhaumik and K. Bhowmik, "A Comparative Assessment of the Performances of Different Edge Detection Operator using Harris Corner Detection Method", International Journal of Computer Applications, Vol. 59, No.19, pp. 7-13 (2012).

6. Z. Ye, Y. Pei and J. Shi, "An Improved Algorithm for Harris Corner Detection", International Congress on Image and Signal Processing (2009). https://doi.org/10.1109/CISP.2009.5304635.

7. Z. Jin-ping, L. Yong-xian, J. Chun-wang, G. Da-meng and L. Jie, "An Improved Harris Corner Distraction Method Based on B_spline", IEEE International Conference on Information Management and Engineering, pp. 504-506 (2010). https://doi.org/10.1109/ICIME.2010.5478127.

8. C. Szegedy, W. Liu, Y. Jia, P. Sermanet, S. Reed, D. Anguelov, D. Erhan, V. Vanhoucke et al., "Going Deeper with Convolutions", Conference on Computer Vision and Pattern Recognition, pp. 1-9 (2015). https://doi.org/10.1109/CVPR.2015.7298594.

9. C. Szegedy, V. Vanhoucke, S. Ioffe and J. Shlens, "Rethinking the Inception Architecture for Computer Vision", Conference on Computer Vision and Pattern Recognition, pp. 28182826 (2016). https://doi.org/10.1109/CVPR.2016.308.

10. Q. Guan, X. Wan, H. Lu, B. Ping, D. Li, L. Wang, Y. Zhu, Y. Wang et al., "Deep convolutional neural network Inception-v3 model for differential diagnosing of lymph node in cytological images: a pilot study", Annals of Translational Medicine, Vol. 7, No. 14, pp. 1-9 (2019). https://doi.org/10.21037/atm.2019.06.29.

11. L. Jiao, H. Wu, R. Bie, A. Umek and A. Kos, "Multisensor Golf Swing Classification Using Deep CNN", Procedia Computer Science, Vol. 129, pp. 59-65 (2018). https://doi.org/10.1016/j.procs.2018.03.046.

12. N. Chouhan, A. Khan and H. Khan, 'Network anomaly detection using channel boosted and residual learning based deep convolutional neural network", Applied Soft Computing, Vol. 83, pp. 1-32 (2019). https://doi.org/10.1016/j.asoc.2019.105612.

13. J. Son, S. J. Park and K. H. Jung, "Towards Accurate Segmentation of Retinal Vessels and the Optic Disc in Fundoscopic Images with Generative Adversarial Networks", Journal of Digital Imaging, pp. 1-14 (2018). https://doi.org/10.1007/s10278-018-0126-3. 